\title{
Clinical profile and outcome among patients with acute-on-chronic liver failure admitted in the intensive care unit
}

\author{
Marwa Lotfi El Sayed ${ }^{1}$, Tarek El Sayed Gouda², E. L. Sayed Abdel Maksood Khalil², \\ Mohammed Mohammed El Sayed Al Arman $^{3}$ and Islam Eid Mohamed ${ }^{2^{*}}$ (i)
}

\begin{abstract}
Background: Acute-on-chronic liver failure (ACLF) has been recently defined as a clinical form including acute hepatic decompensation and high 28-day mortality. ACLF usually follows a precipitating event on the background of established cirrhosis. ACLF is considered the most frequent indication for admission to the ICU among cirrhotic patients. Our research aimed to reveal the clinical profile and outcome among patients with ACLF to detect an allocation system of these patients to the intensive care unit (ICU), and a decision tool for clinical practice. It is a prospective study of 60 patients with ACLF. Patients are divided into group A that included 30 patients with ACLF admitted to the hepatology and gastroenterology ward and group B that also included 30 patients with ACLF admitted to the ICU. Each group is subdivided into subgroups regarding the grade of ACLF.

Results: The most common precipitating factor of ACLF is SBP 78.3\% (80\% in ICU, 73.6\% inward). Renal failure is the most common organ failure in ACLF in both groups. CLIF-C ACLF is assumed to be a highly prognostic score for mortality in ACLF patients better than other scores. ROC curve of CLIF-C ACLF with AUC: 0.972 and Cl: 0.919, 1.025 showed a cutoff point $=57.0$ above which intensive care admission does not seem to benefit ACLF patients. The sensitivity at the optimal cut point is $88.89 \%$ and the specificity is $100 \%$. There is a significant difference between the 3 ACLF groups regarding 1-month and 3-month mortalities in patients admitted to the ICU. ACLF1 shows the least 1month and 3-month mortality rates while ACLF3 shows the highest mortality rates in ICU patients ((1-month mortality: 20\%,60\%, 100\% in ACLF1, 2, 3 respectively), (3-month mortality: 50\%, 80\%, 100\% in ACLF1, 2, 3 respectively)).
\end{abstract}

Conclusion: Mortality is high in ACLF and increases with the number of organ failures (40\% in ACLF1 to 100\% in ACLF3). CLIFC-ACLF is the most prognostic scoring system with a cut-off value of 57; above this value, mortality is a fact.

Keywords: ACLF, CLIF-C ACLF, ICU, 1-month mortality, 3-month mortality

\footnotetext{
*Correspondence: Islameedmuh4@yahoo.com

${ }^{2}$ Department of Internal Medicine, Faculty of Medicine, Mansoura University,

Mansoura 35511, Egypt

Full list of author information is available at the end of the article
}

\section{Springer Open}

(c) The Author(s). 2021 Open Access This article is licensed under a Creative Commons Attribution 4.0 International License, which permits use, sharing, adaptation, distribution and reproduction in any medium or format, as long as you give appropriate credit to the original author(s) and the source, provide a link to the Creative Commons licence, and indicate if changes were made. The images or other third party material in this article are included in the article's Creative Commons licence, unless indicated otherwise in a credit line to the material. If material is not included in the article's Creative Commons licence and your intended use is not permitted by statutory regulation or exceeds the permitted use, you will need to obtain permission directly from the copyright holder. To view a copy of this licence, visit http://creativecommons.org/licenses/by/4.0/. 


\section{Background}

Acute-on-chronic liver failure (ACLF) is a clinical syndrome of sudden hepatic decompensation in patients with pre-existing chronic liver disease that is associated with one or more extra-hepatic organ failures and increased mortality [1]. ACLF is characterized by a rapidly deteriorating course in a previously diagnosed or undiagnosed chronic liver disease with a potential for reversibility [2].

The European Association for the Study of the Liver-Chronic Liver Failure (EASL-CLIF) Consortium studied and defined the grades of ACLF in the CANONIC study [3].

The exact pathogenesis of ACLF is still confusing; it usually results from a provoking factor and disturbance in the host response to injury [4]. The etiology of ACLF would be a precipitating event on the background of established cirrhosis. Both the precipitating event and the pre-existing liver disease have geographical disparities. These events could be either liver-related (superimposed viral hepatitis, alcoholic hepatitis, portal vein thrombosis (PVT), drug-induced liver injury (DILI)), or non-liver-related (surgery, infections, trauma). There is no specific precipitating event to be found in about $40 \%$ of patients with ACLF as well [5]. ACLF is a dynamic syndrome, which may improve, worsen, or have a mild protracted course allowing us to evaluate for a possible liver transplant. The etiology of the precipitating factor causing ACLF does not alter the prognosis [6].

Systemic inflammation is a hallmark of ACLF. That is why white cell count and plasma levels of C-reactive protein (CRP) and pro-inflammatory molecules such as interleukin (IL)-6, IL-1 $\beta$, IL-8 are higher in patients with ACLF than in those without $[7,8]$. The pathogenesis of ACLF is intrinsically linked to the abnormal host response to precipitating injury and SIRS, and the prognosis of the patient is chiefly based upon the degree of immune paresis and severity of organ failure [9].

In general, patients with two or more extra-hepatic organ failures have a high mortality risk. Respiratory failure is the strongest predictor of death. Patients with infection-related ACLF have the high opportunity to delist from liver transplant (42\%) [10]. Two categories of prognostic models have been used: first, those evaluating the severity of liver disease and, second, those evaluating the dysfunction of several organ systems. It has been shown that liver function is not the main determinant of clinical outcome for patients with decompensated cirrhosis; thus, liver-specific scoring systems, such as the CTP or the MELD score, have limitations in accurately predicting the outcome of patients with ACLF. Organ failure scores, such as the APACHE II and III and SOFA score CLIF-SOFA, are more helpful in predicting survival [11].
Prognosis is associated with types and number of failed organs and this can be measured by the chronic liver failure (CLIF) organ failure (CLIF-OF) score. The CLIF-C ACLF score adds age and leukocytic count as variables that increase the predictive accuracy. The survival is considered zero in patients with a CLIF-C ACLF score $>64$ without urgent liver transplant [12].

Therefore, in our research, we studied the clinical and biochemical profiles, etiology, and outcome of ACLF patients.

\section{Methods}

It is a prospective study that included 60 patients with acute-on-chronic liver failure. All involved cases were with liver cirrhosis. The definitions of organ failures were established using (CLIF-SOFA) score (renal failure: serum creatinine $\geq 2.0 \mathrm{mg} / \mathrm{dL}$ or need for renal replacement therapy, liver failure: serum bilirubin level $\geq$ of $12.0 \mathrm{mg} / \mathrm{dL}$, cerebral failure: grade III or IV hepatic encephalopathy, coagulation failure: either INR $>2.5$ and/or platelet count $\leq 20 \times 10^{9} / \mathrm{L}$, circulatory failure: use of vasopressors such as dopamine or terlipressin, respiratory failure: $\mathrm{PaO} 2 /$ $\mathrm{FiO} 2 \leq 200$ or $\mathrm{SpO} 2 / \mathrm{FiO} 2 \leq 200$.

Patients were graded into three grades according to the number of failed organs:

- ACLF grade1: This group includes 3 subgroups of patients:

1. Single kidney failure

2. Single failure of the liver, coagulation, circulation, or respiration and serum creatinine 1.5 to $1.9 \mathrm{mg} / \mathrm{d}$ or grade 1 or 2 hepatic encephalopathy

3. Cerebral failure (HE grades 3 or 4 ) with serum creatinine range from 1.5 to $1.9 \mathrm{mg} / \mathrm{dL}$.

- ACLF grade 2: It includes patients who have two failed organs.

- ACLF grade 3: It includes patients who have 3 or more failed organs.

Exclusion criteria: age $<18$ and $>80$, known cases of hepatocellular carcinoma who underwent treatment, metastatic liver disease in the otherwise non-cirrhotic liver, cholangiocarcinoma in the non-cirrhotic liver, fulminant liver failure, acute viral hepatitis in a previous non-cirrhotic patient, drug-induced liver injury in a previous non-cirrhotic patient, HIV/AIDS, pregnant women, patients with any disseminated malignancy.

The involved patients in the study were classified into two main groups: 
Group A: This included 30 patients with ACLF admitted to the hepatology and gastroenterology ward. Group B: This included 30 patients with ACLF admitted to the hepatology and gastroenterology ICU.

All patients were subjected to the following: thorough history taking, complete physical examination, laboratory investigations: biochemical liver tests, serum creatinine, complete blood count $(\mathrm{CBC})$, serum electrolyte $(\mathrm{Na}, \mathrm{K})$, $\mathrm{C}$-reactive protein, ascitic fluid analysis, urine analysis, virology markers (HAV Ig M antibody and HEV Ig M antibody for patients with a 3-fold increase in liver enzymes, quantitative PCR for $\mathrm{HCV}, \mathrm{HBsAg}$, anti-HBc IgG, and in some cases HBV DNA PCR), and radiological studies: abdominal ultrasound and Chest X-ray.

Prognostic scores were calculated in all involved cases: Child Turcotte Pugh (CTP), Model of End-Stage Liver Disease (MELD), Model of End-Stage Liver Disease Na (MELD-Na), APACHE II SCORE, Sequential Organ Failure Assessment score (SOFA score), Chronic liver failure (CLIF)-SOFA, CLIF Consortium Organ Failure Score (CLIF-C-OFs), and CLIF-C-ACLF score.

\section{Statistical analysis}

All data was fed to statistical analysis using R Software version 3.5.2 (2018-12-20)-"Eggshell Igloo," and the appropriate statistical tests have been carried out. The statistical analysis was based on a two-tailed test using a level of significance for analysis at $P$-value $\leq 0.05$.

\section{Results}

Our study involved 60 patients with ACLF (30 patients admitted to the ward and 30 patients admitted to ICU).
Patients in the ICU were classified according to ACLF grades into 3 subgrades (ACLF1, ACLF2, and ACLF3). Each subgrade involved 10 patients (33.3\%)). Patients in the ward were classified according to ACLF grades into 3 grades (ACLF1, ACLF2, and ACLF3). Each subgrade involved 10 patients (33.3\%).

There is a non-significant difference between the patients of the two main groups regarding age, sex, and geographic area distribution. There is a nonsignificant difference in the etiology of chronic liver diseases between the ward and ICU groups. HCV is the main cause in ICU $((N=27) 90 \%)$ and ward $((N$ = 29) $96.7 \%)$ groups. The second etiology is hepatitis B viral infection (ICU group $((N=3) 10 \%)$, ward group $(N=1) 3.3 \%)$.

The most common precipitating factor of ACLF is a bacterial infection in both ward (23 patients $73.6 \%$ ) and ICU (24 patients 80\%). The most common precipitating factor is SBP in ICU $(N=21(70 \%))$ and ward groups $(N=19(63.3 \%))$. Chest infection (1 patient in ICU $3.3 \%, 3$ patients in ward 10\%) is the second etiology of sepsis in our study. The second precipitating factor of ACLF in our study is upper gastrointestinal bleeding which represents (5 patients in ICU 16.7\%, 3 patients in ward 10\%). Large-volume paracentesis without albumin is also a precipitating factor (1 patient in ward 3.3\%, 1 patient in ICU 3.3\%) (Fig. 1).

We found that the most common organ failure in ACLF is renal failure as it represents ( 25 patients in ward $83.8 \%, 18$ patients in ICU 60\%). The second common organ failure in this study was cerebral failure ( 8 patients in the ward $(26.7 \%, 23$ patients in the

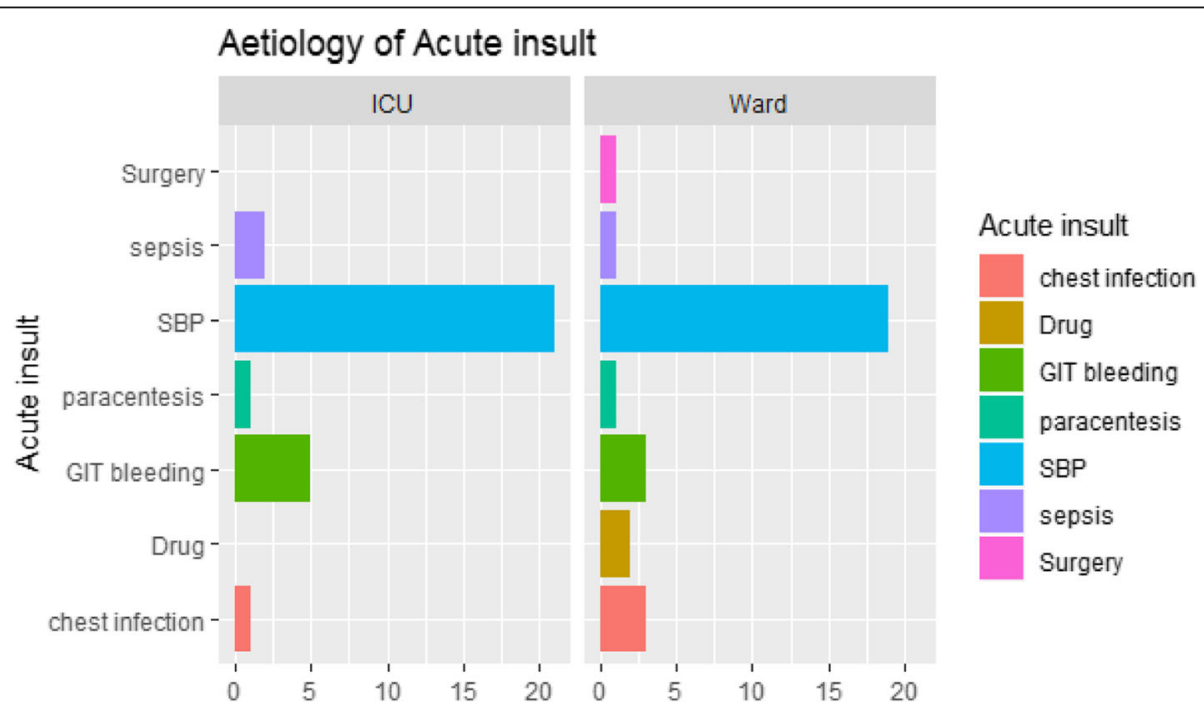

Fig. 1 Precipitating factor (acute insult) of ACLF. Figure 1 shows a non-significant difference in the Precipitating factor (acute insult) between ICU and ward patients (P-value $>0.05)$. The most common precipitating factor is SBP in ICU and ward groups 
ICU 76.7\%). Cerebral and circulatory failures are more common in ICU patients $(76.7 \%$ and $33.3 \%$ respectively) while coagulopathy is more common in ward patients $(43.3 \%)$ (Table 1$)$.

The results showed a significant increase in hepatic encephalopathy and a significant decrease in GCS in subgroups ACLF1, 2, 3 in the ICU compared to subgroups ACLF1, 2, 3 in the ward. In addition, it showed a significant increase in the respiratory rate in ACLF3 in the ICU compared to ACLF3 in the ward (Table 2).

We studied the biochemical profile of ACLF patients admitted to ward and ICU, we found no significant difference between ward patients and ICU patients as regards the hemoglobin level, total leucocytic count, and platelet count. Although the study showed a low hemoglobin level in ACLF patients in both groups (ward with (mean 9.4 $\pm 1.5 \mathrm{SD}$ ) and ICU with (mean $9.9 \pm 1.8$ SD)). It also showed that there was a low platelet count in ACLF patients in the ward and ICU with a mean of $95.7 \pm 62.7$ SD and 106.2 \pm 43.2 SD respectively. The result of this study showed a low serum albumin level in ACLF patients with a mean of $2.6 \pm 0.7 \mathrm{SD}$ in the ward and a mean of $2.2 \pm 0.4 \mathrm{SD}$ in the ICU) with a significant difference between the 2 groups $(P$-value $<0.05)$. The albumin level was decreased more in patients admitted to the ICU (Table 3).

In the present study, there was a higher C-reactive protein level in ACLF patients admitted to ward and ICU with a mean of $67.7 \pm 6.0 \mathrm{SD}$ and $87.8 \pm 28.1 \mathrm{SD}$ respectively with a significant difference between the 2 groups $(P$-value $<0.05)$.

A significant increase in APACHE score in the ICU group was noticed compared to the ward group. CLIF-SOFA, CLIF-C OF, and CLIF-C ACLF are statistically significant, whereas CTP, APACHE, MELD, MELD $\mathrm{Na}^{+}$, and SOFA scores are statistically nonsignificant as regards mortality. CLIF-C ACLF is assumed to be a highly prognostic score as regards mortality in ACLF patients as compared to other scores. The results showed a significant difference between the 3 ACLF groups regarding $1 \mathrm{M}$. and $3 \mathrm{M}$. in the ICU group (Table 4, 5, 6, 7, 8, and 9).

CLIC C-ACLF score ROC curve $($ AUC) $=0.972$ with CI: 0.919, 1.025, cutoff point $=57.0$ above which intensive care admission does not seem to benefit ACLF patients. Optimal sensitivity at the actual cut point is $0.889(88.89 \%)$ and optimal specificity at the actual cut-point is 1.0 (100\%) (Fig. 2). Figures 3 and 4 clarify that there is a significant difference between the no. of organ failure and $1 \mathrm{M}$. and 3M. mortality respectively. Figures 5 and 6 show that there is a non-significant difference between ward and ICU regarding $1 \mathrm{M}, 3 \mathrm{M}$ mortality.

Our study concluded an algorithm to stratify patients according to prognosis is needed, in order to monitor treatment responsiveness, determine emergency for transplantation, and decide allocation in the intensive care unit (ICU) and also to have a rational basis to decide futility.

- Intensive care candidate (1): ACLF grade $<2$ with the need for intensive therapy, e.g., $\mathrm{HE}>2$ or respiratory insufficiency (2), CLIF-C ACLF $<57$.

- Possible intensive care candidate, eligibility should be considered and discussed with ICU (1): ACLF grade 2 with CLIF-C ACLF $<57$ if continuous organ failure is present after 3 days of complete intensive care treatment, the outcome is questionable.

- Unlikely to benefit from intensive care therapy cases can be discussed, e.g., patients that are candidates for liver transplantation (1): ACLF grade 3 (2), CLIF-C ACLF > 57 .

\section{Discussion}

The main etiology of chronic liver disease in our study is Hepatitis C viral infection (HCV 27 patients admitted to ICU, 29 patients admitted to ward) as Hepatitis $\mathrm{C}$ viral infection is endemic in Egypt with the highest prevalence rate in the world $[13,14]$.

Table 1 Types of organ failure on admission in the studied groups

\begin{tabular}{|c|c|c|c|c|c|}
\hline & \multicolumn{2}{|c|}{ Ward group } & \multicolumn{2}{|c|}{ ICU group } & \multirow{2}{*}{$\begin{array}{l}P \text { - } \\
\text { value }\end{array}$} \\
\hline & $N$ & $\%$ & $N$ & $\%$ & \\
\hline Cerebral F. & 8 & 26.7 & 23 & 76.7 & 0.0003 \\
\hline Renal F. & 25 & 83.3 & 18 & 60.0 & 0.086 \\
\hline Circulatory F. & 1 & 3.3 & 10 & 33.3 & 0.0076 \\
\hline Hepatic F. & 9 & 30.0 & 4 & 13.3 & 0.21 \\
\hline Respiratory F. & 8 & 26.7 & 14 & 46.7 & 0.18 \\
\hline Coagulation F. & 13 & 43.3 & 5 & 16.7 & 0.049 \\
\hline
\end{tabular}

Table 1 revealed that cerebral, coagulation, and circulatory failure showed a significant difference between the ward and ICU groups. Cerebral and circulatory failures are more common in ICU patients while coagulopathy is more common in ward patients. Renal failure is the most common organ failure in ACLF in both groups 


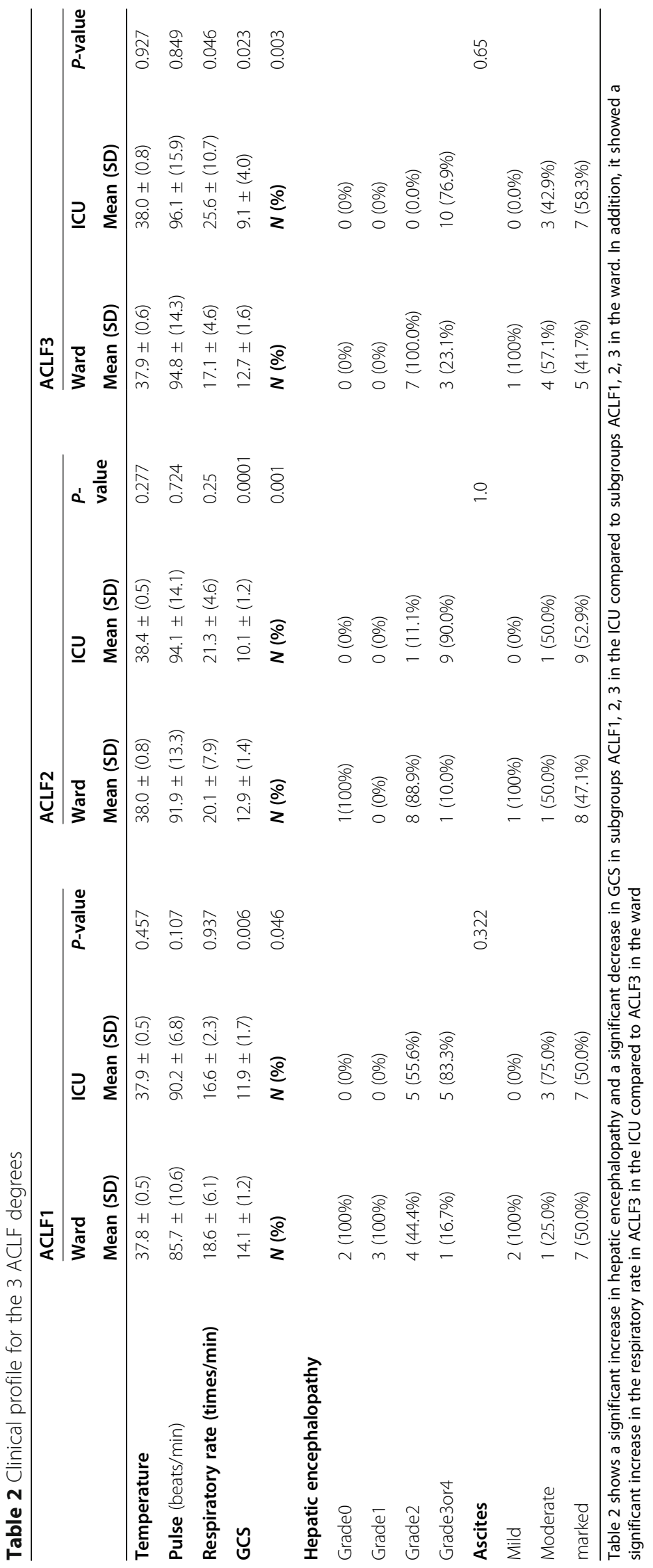




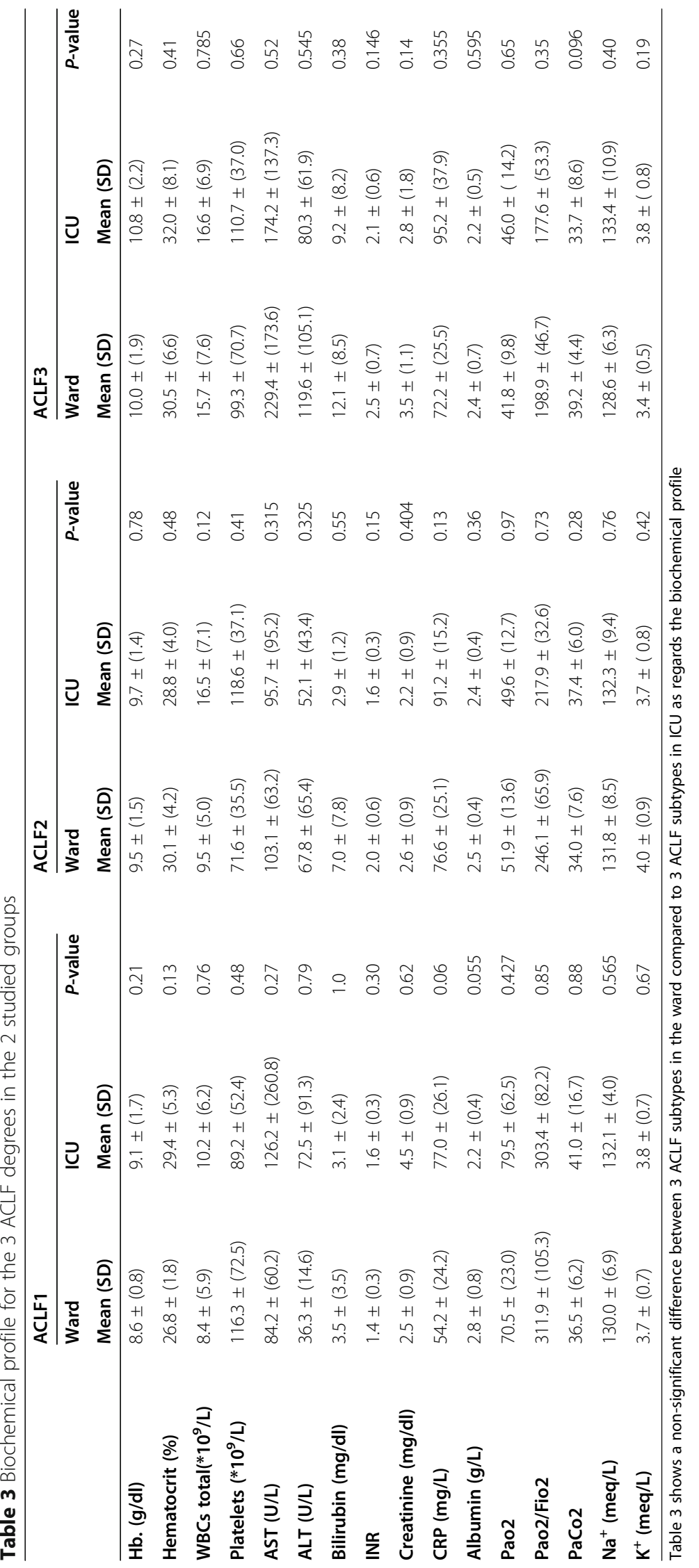


Table 4 Prognostic scores of the 2 studied groups

\begin{tabular}{llll}
\hline & Ward & ICU & P-value \\
& Mean (SD) & Mean (SD) & N (\%) \\
\hline CTP score & & & 0.232 \\
Child A & $0(0.0 \%)$ & $0(0.0 \%)$ & $2(6.7 \%)$ \\
Child B & $5(16.7 \%)$ & $28(93.3 \%)$ & $24.5 \pm(5.8)$ \\
Child C & $25(83.3 \%)$ & $26.6 \pm(5.6)$ & 0.074 \\
MELD & $28.3 \pm(8.2)$ & $18.3 \pm(5.5)$ & 0.052 \\
MELD Na ${ }^{+}$ & $30.5 \pm(6.7)$ & $10.1 \pm(3.1)$ & 0.03 \\
APACHE & $15.5 \pm(4.1)$ & $9.2 \pm(3.2)$ & 0.32 \\
SOFA & $9.1 \pm(2.5)$ & $11.4 \pm(2.7)$ & 0.54 \\
CLIF SOFA & $8.5 \pm(2.6)$ & $56.5 \pm(11.3)$ & 0.92 \\
CLIF. OF & $11.1 \pm(2.2)$ & $55.1 \pm(9.9)$ & 0.61 \\
CLIF C ACLF & & & \\
\hline
\end{tabular}

Table 4 shows a non-significant difference in CTP score, MELD, MELD Na ${ }^{+}$, SOFA, CLIF-SOFA, CLIF-C OF, and CLIF-C ACLF between the ward group compared to the ICU group, but showed a significant increase in APACHE in the ICU group compared to the ward group

Table 5 Prognostic scores for the 3 ACLF degrees in both ward and ICU patients

\begin{tabular}{|c|c|c|c|c|c|c|c|}
\hline & \multicolumn{2}{|l|}{ ACLF1 } & \multicolumn{2}{|l|}{ ACLF2 } & \multicolumn{2}{|l|}{ ACLF3 } & \multirow[t]{3}{*}{$P$-value } \\
\hline & Ward & ICU & Ward & ICU & Ward & ICU & \\
\hline & $N(\%)$ & $N(\%)$ & $N(\%)$ & $N(\%)$ & $N(\%)$ & $N(\%)$ & \\
\hline CTP Score & & & & & & & 0.0003 \\
\hline Child A & $1(5 \%)$ & $0(0 \%)$ & $0(0 \%)$ & $0(0 \%)$ & $0(0 \%)$ & $0(0 \%)$ & \\
\hline Child B & $4(20 \%)$ & $1(5 \%)$ & $1(5 \%)$ & $1(5 \%)$ & $0(0 \%)$ & $0(0 \%)$ & \\
\hline Child C & $5(25 \%)$ & $9(45 \%)$ & $9(45 \%)$ & $9(45 \%)$ & 10 (50\%) & 10 (50\%) & \\
\hline \multirow[t]{2}{*}{$P$-value } & 0.14 & & 1.0 & & 1.0 & & \\
\hline & Mean (SD) & Mean (SD) & Mean (SD) & Mean (SD) & Mean (SD) & Mean (SD) & \\
\hline MELD & $21.5 \pm(3.5)$ & $21.9 \pm(3.7)$ & $28.2 \pm(6.3)$ & $22.5 \pm(4.4)$ & $35.1 \pm(7.7)$ & $29.1 \pm(6.5)$ & $8.2 \times 10^{-5}$ \\
\hline$P$-value & 0.81 & & 0.043 & & 0.076 & & \\
\hline MELD Na ${ }^{+}$ & $25.0 \pm(3.1)$ & $24.2 \pm(3.6)$ & $31.1 \pm(4.9)$ & $25.6 \pm(4.6)$ & $35.4 \pm(7.0)$ & $29.9 \pm(7.0)$ & 0.001 \\
\hline$P$-value & 0.598 & & 0.119 & & 0.129 & & \\
\hline APACHE & $12.1 \pm(4.2)$ & $13.6 \pm(4.2)$ & $16.1 \pm(2.2)$ & $19.8 \pm(5.3)$ & $18.3 \pm(3.0)$ & $21.5 \pm(3.7)$ & $3.4 \times 10^{-6}$ \\
\hline$P$-value & 0.433 & & 0.066 & & 0.0495 & & \\
\hline SOFA & $6.9 \pm(1.7)$ & $8.4 \pm(1.9)$ & $9.5 \pm(1.4)$ & $8.7 \pm(1.6)$ & $11.0 \pm(2.5)$ & $13.2 \pm(3.0)$ & $3.5 \times 10^{-6}$ \\
\hline$P$-value & 0.081 & & 0.25 & & 0.093 & & \\
\hline CLIF SOFA & $6.0 \pm(1.3)$ & $6.8 \pm(1.4)$ & $8.3 \pm(1.3)$ & $8.2 \pm(1.4)$ & $11.2 \pm(1.7)$ & $13.0 \pm(2.4)$ & $1.4 \times 10^{-9}$ \\
\hline$P$-value & 0.21 & & 0.872 & & 0.086 & & \\
\hline CLIF. OF & $8.9 \pm(0.6)$ & $9.2 \pm(0.8)$ & $10.7 \pm(0.5)$ & $10.3 \pm(0.5)$ & $13.6 \pm(1.5)$ & $14.6 \pm(2.2)$ & $2.3 \times 10^{-11}$ \\
\hline$P$-value & 0.335 & & 0.089 & & 0.256 & & \\
\hline CLIF C ACLF & $45.5 \pm(6.3)$ & $46.1 \pm(7.1)$ & $54.3 \pm(4.9)$ & $54.7 \pm(5.7)$ & $65.5 \pm(5.0)$ & $68.7 \pm(6.5)$ & $1.6 \times 10^{-15}$ \\
\hline$P$-value & 0.844 & & 0.868 & & 0.238 & & \\
\hline
\end{tabular}

Table 5 shows a significant increase in all prognostic scores with increasing the grade of ACLF in all patients in the ward and ICU and also showed a nonsignificant difference between ACLF subgroups in the ward compared to the ICU group except for APACHE that showed a significant increase in subgroup ACLF3 in the ICU compared to subgroup ACLF3 in the ward 
Table 6 Prognostic scores on admission and mortality

\begin{tabular}{|c|c|c|c|}
\hline \multirow{3}{*}{$\begin{array}{l}\text { Prognostic } \\
\text { score }\end{array}$} & \multicolumn{2}{|l|}{ Mortality } & \multirow[t]{3}{*}{$P$-value } \\
\hline & Death & Survival & \\
\hline & Count (\%) & Count (\%) & \\
\hline СТP & & & 0.634 \\
\hline Child A & $0(0.0 \%)$ & $0(0.0 \%)$ & \\
\hline Child B & 7 (100.0\%) & 0 (0.0\%) & \\
\hline \multirow[t]{2}{*}{ Child C } & 45 (86.5\%) & 8 (13.5\%) & \\
\hline & Mean (SD) & Mean (SD) & \\
\hline MELD & $27.0(7.5)$ & $22.4(4.0)$ & 0.107 \\
\hline MELD Na ${ }^{+}$ & 29.1 (6.6) & $24.8(4.0)$ & 0.088 \\
\hline APACHE & $17.4(4.8)$ & $13.9(5.5)$ & 0.07 \\
\hline SOFA & 9.9 (2.9) & 7.9 (1.9) & 0.07 \\
\hline CLIF-SOFA & $9.2(2.9)$ & $6.8(1.5)$ & 0.034 \\
\hline CLIF-C OF & $11.5(2.5)$ & $9.4(0.7)$ & 0.0378 \\
\hline CLIF-C ACLF & $57.5(10.1)$ & $44.6(6.1)$ & 0.005 \\
\hline
\end{tabular}

In our study, the most common precipitating factor of ACLF is bacterial infection in both ward and ICU. This agrees with the study of Fernandez and colleagues [15], $37 \%$ of patients with ACLF had a bacterial infection at the time of ACLF diagnosis, and Barosa and colleagues [16] who found $40.7 \%$ of ACLF was precipitated by infection.

The most common type of infection found in this study is SBP in both ward and ICU. This result agrees with Hernaez and colleagues [17] who found that SBP is the most common cause of sepsis-induced ACLF.

Chest infection is a common cause of hepatic decompensation and considered a risk factor for mortality and this agrees with our study [18-20].

Table 7 Relation between prognostic scores and the detected mortality

\begin{tabular}{lll}
\hline Prognostic scores & $\begin{array}{l}\text { Adjusted } P \text {-value } \\
\text { regarding mortality }\end{array}$ & Test of significance \\
\hline CTP & 0.28 & $\begin{array}{l}\text { Logistic regression } \\
\text { model }\end{array}$ \\
MELD & 0.42 & \\
MELD-Na ${ }^{+}$ & 0.36 & \\
APACHE & 0.25 & \\
SOFA & 0.46 & \\
CLIF.SOFA & 0.95 & \\
CLIF.OF & 0.77 & \\
CLIF-C ACLF & 0.048 &
\end{tabular}

Table 7 shows that the adjusted $P$-value as regards mortality of CLIF-C ACLF as multi-various (in presence of other scores) was statistically significant. So, CLIFC ACLF is assumed to be a highly prognostic score as regards mortality in ACLF patients as compared to other scores
The second precipitating factor in our study is upper gastrointestinal bleeding. This agrees with the CANONIC study as this factor represents $13.8 \%$ [3]. In our study, large-volume paracentesis without albumin is also a precipitating factor. This agrees with the CANONIC study in 2012 and Shi with his colleagues [3, 21].

We found that the most common organ failure in ACLF is renal failure. This result agrees with the CANONIC study [3].

We found no significant difference between ward patients and ICU patients as regards hemoglobin level, total leucocytic count, and platelet count. Although our study showed a low hemoglobin level in ACLF patients in both groups. This result agrees with Piano and colleagues' 2017 study that showed low values of hemoglobin in patients with ACLF [22]. Our study showed that there was a low platelet count in ACLF patients in the ward and ICU; this agrees with Zhang and colleagues' 2016 study [23].

The result of this study showed a low serum albumin level in ACLF patients with a significant difference

Table 8 Mortality between 3 ACLF groups in ward

\begin{tabular}{|c|c|c|c|c|c|c|c|}
\hline & \multicolumn{2}{|c|}{ ACLF1 } & \multicolumn{2}{|c|}{ ACLF2 } & \multicolumn{2}{|c|}{ ACLF3 } & \multirow[t]{2}{*}{$P$-value } \\
\hline & $N$ & $\%$ & $N$ & $\%$ & $N$ & $\%$ & \\
\hline 1M. mortality & 6 & $60 \%$ & 8 & $80 \%$ & 10 & $100 \%$ & 0.122 \\
\hline 3M. mortality & 9 & $90 \%$ & 10 & $100 \%$ & 10 & $100 \%$ & 1.0 \\
\hline
\end{tabular}


Table 9 Mortality between 3 ACLF groups in ICU

\begin{tabular}{|c|c|c|c|c|c|c|c|}
\hline & \multicolumn{2}{|c|}{ ACLF1 } & \multicolumn{2}{|c|}{ ACLF2 } & \multicolumn{2}{|c|}{ ACLF3 } & \multirow{2}{*}{$\begin{array}{l}P \text { - } \\
\text { value }\end{array}$} \\
\hline & $\bar{N}$ & $\%$ & $N$ & $\%$ & $N$ & $\%$ & \\
\hline 1M. mortality & 2 & $20 \%$ & 6 & $60 \%$ & 10 & $100 \%$ & 0.001 \\
\hline 3M. mortality & 5 & $50 \%$ & 8 & $80 \%$ & 10 & $100 \%$ & 0.04 \\
\hline
\end{tabular}

Table 9 shows that there is a significant difference between the 3 ACLF groups regarding $1 \mathrm{M}$. and $3 \mathrm{M}$. mortalities in patients admitted the ICU. ACLF1 showed the least mortality rates while ACLF3 showed the highest mortality rates in ICU patients. $1 \mathrm{M}$. mortality was $20 \%, 60 \%, 100 \%$ in ACLF1, 2, 3 respectively and $3 \mathrm{~m}$ mortality was $50 \%, 80 \%, 100 \%$ in ACLF1, 2, 3 respectively

between the 2 groups. The albumin level was decreased more in patients admitted to ICU; this agrees with Ferrarese and his colleagues 2019 [24] who found a significant difference in albumin level between ward and ICU.

In the present study, there was a higher C-reactive protein level in ACLF patients admitted to ward and ICU with a significant difference between the 2 groups. This runs parallel to Cervoni and his colleagues' 2012 study [25] and Pieri and his colleagues' 2014 study [26] which found that persistently elevated CRP in ACLF patients can help to identify patients with a higher shortterm mortality risk.

In our study, we found that CLIF-C ACLF was the best prognostic model as regards mortality, whereas CTP, APACHE, MELD, MELD $\mathrm{Na}^{+}$, and SOFA scores were statistically non-significant. This result agrees with Chen and his colleagues' 2020 study [27] who found that the CLIF-C ACLF score was significantly superior to other models in predicting overall mortality.

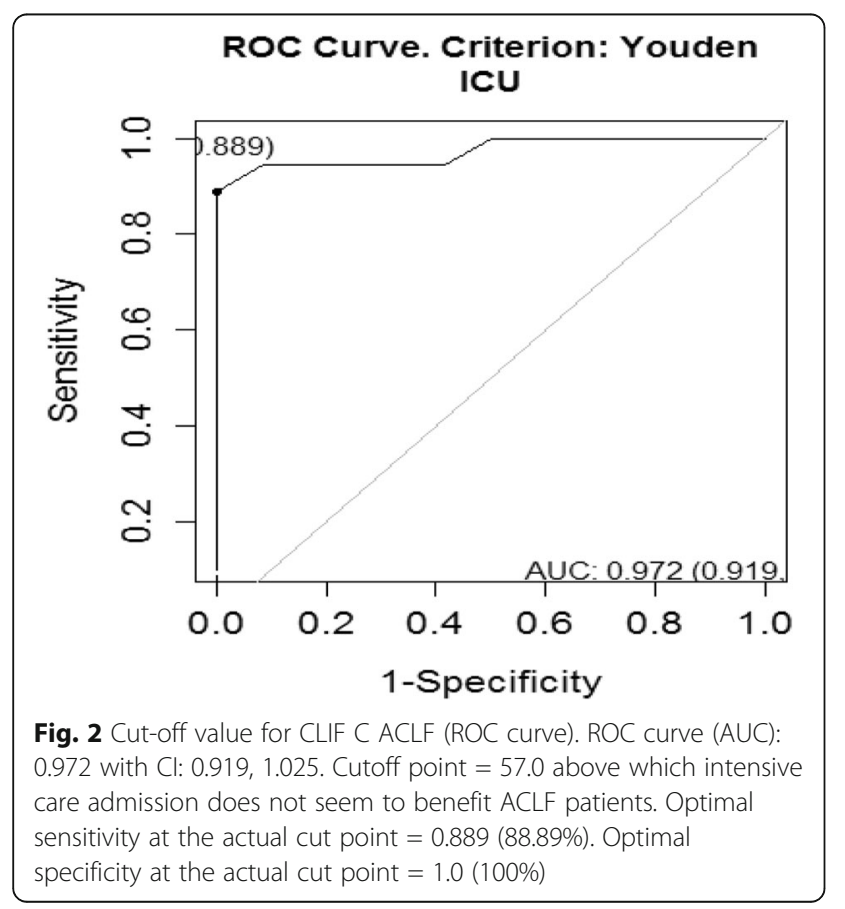

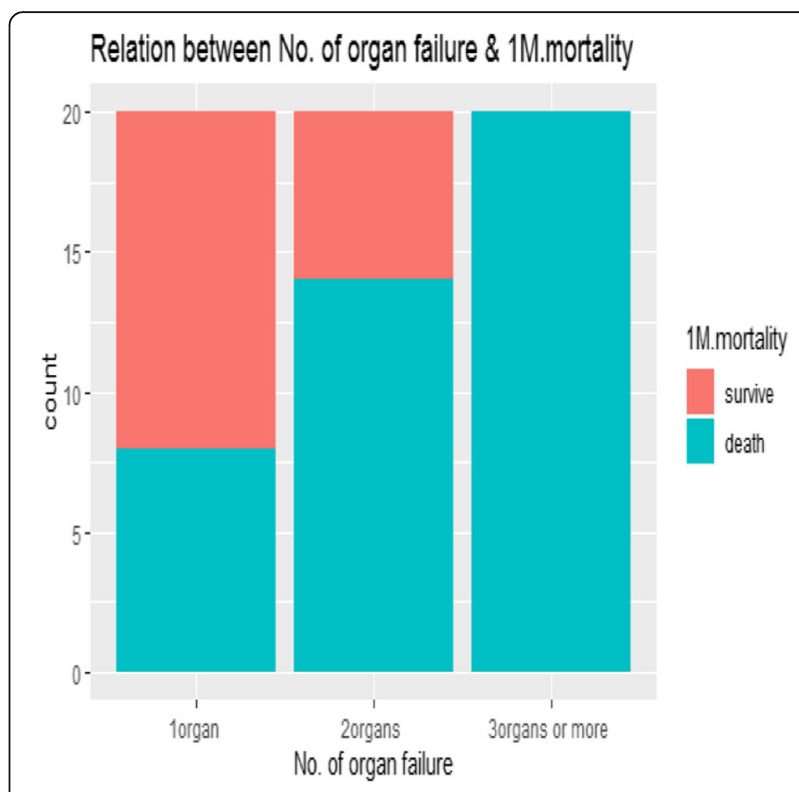

Fig. 3 Relation between no. of organ failure and 1M. mortality. Figures 3 and 4 show that there is a significant difference between no. of organ failure and both $1 \mathrm{M}$. and $3 \mathrm{M}$. mortalities

We studied CLIC-C ACLF score ROC curve (AUC) $=0.972$ with CI: $0.919,1.025$, cutoff point $=57$ above which intensive care admission does not seem to benefit ACLF patients; this runs with Engelmann and his colleagues' 2018 study [28] who also examined the CLIF-C ACLF score. All the patients with a CLIF-C

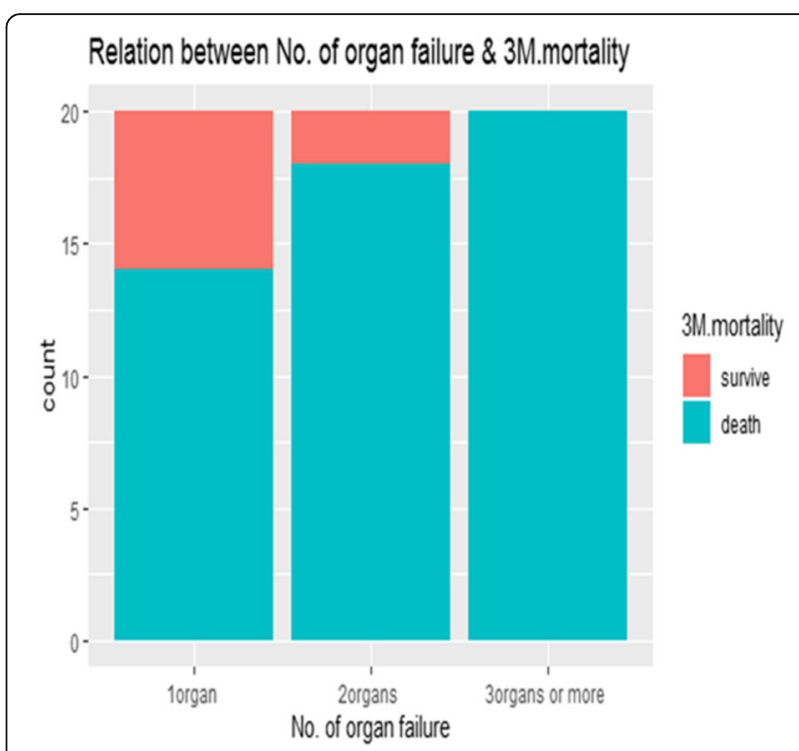

Fig. 4 Relation between no. of organ failure 3M. mortality. Figures 3 and 4 show that there is a significant difference between no. of organ failure and both $1 \mathrm{M}$. and $3 \mathrm{M}$. mortalities 


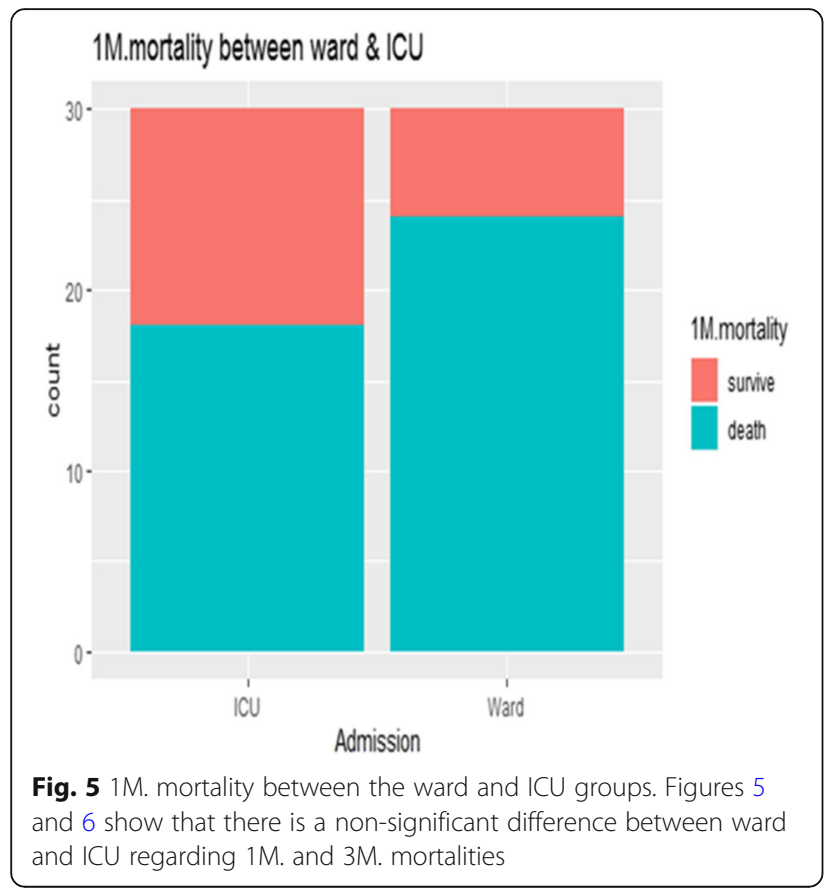

ACLF score greater than 70 (on admission or 48 hours post-ICU admission) died within 28 days [28]. Ramzan and colleagues' 2020 study concluded that a CLIF-C ACLF score $\geq 70$ at $48 \mathrm{~h}$ and organ failure are better predictors of mortality, and also, ICU care in those patients does not benefit them. The definitive therapy with liver transplantation may have a promising role, if it is considered early [29].

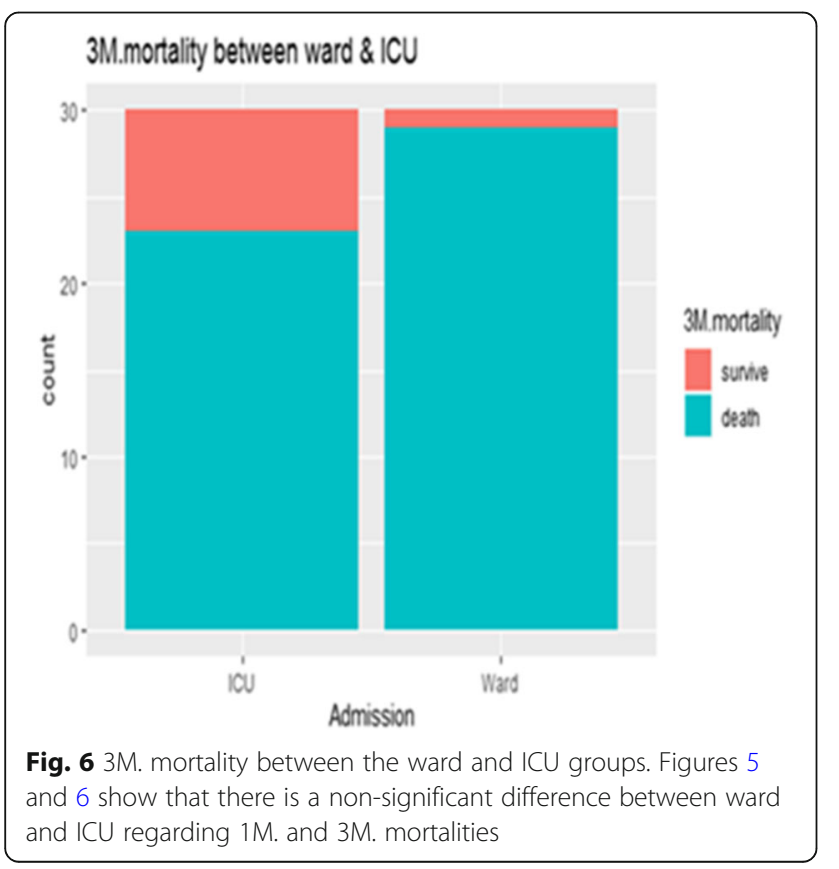

\section{Conclusion}

ACLF is a highly dynamic issue in cirrhotic patients. Mortality is high in ACLF and increases with the number of organ failures (40\% in ACLF1 to 100\% in ACLF3). CLIFC-ACLF is the most prognostic scoring system with a cutoff value of 57 , above this value mortality is a fact.

\section{Abbreviations \\ CLIF-C ACLF: Chronic liver failure consortium acute on chronic liver failure; SOFA score: Sequential Organ Failure Assessment score; APACHE \\ score: Acute Physiologic Assessment and Chronic Health Evaluation score}

\section{Authors' contributions}

$\mathrm{ML}$ and EE made practical work (collection of data and patients follow-up). EE made statistical analysis and interpretation of results and paper revision. TE made the main research idea, initial paper structure, and paper revision. EA made the initial paper structure and paper revision. MM made the analysis of pathological data. All authors have read and approved the manuscript.

\section{Availability of data and materials}

The data used and/or analyzed during the current study are available from the corresponding author on reasonable request.

\section{Declarations}

\section{Ethics approval and consent to participate}

The study protocol was approved by the Ethics Review Board of Faculty of Medicine, Mansoura University, and informed written consent was obtained from all participants according to the Declaration of Helsinki. The ethical committee's reference number is MD/17.06.106. The date of ethical committee approval: 17th July 2017.

\section{Consent for publication}

Written consent was obtained from the patients to participate in the study and to share and publish its findings.

\section{Competing interests}

The authors declare that they have no competing interests.

\section{Author details}

${ }^{1}$ General Senbellawein Hospital, Ministry of Health, El-Senbellawein, Egypt. ${ }^{2}$ Department of Internal Medicine, Faculty of Medicine, Mansoura University, Mansoura 35511, Egypt. ${ }^{3}$ Department of Clinical Pathology, Faculty of Medicine, Mansoura University, Mansoura 35511, Egypt.

Received: 10 May 2021 Accepted: 28 July 2021

Published online: 22 September 2021

\section{References}

1. Arroyo V, Moreau R, Jalan R (2020) Acute-on-chronic liver failure. N Engl J Med 382(22):2137-2145. https://doi.org/10.1056/NEJMra1914900

2. Kumar BR, Rahul D, Prabhakar B (2016) A study of clinical profile in patients with acute on chronic liver failure in a tertiary hospital. Asian Pac J Health Sci 3(2):47-57. https://doi.org/10.21276/apjhs.2016.3.2.10

3. Moreau R, Jalan R, Gines P, Pavesi M, Angeli P, Cordoba J, Durand F, Gustot T, Saliba F, Domenicali M, Gerbes A, Wendon J, Alessandria C, Laleman W, Zeuzem S, Trebicka J, Bernardi M, Arroyo V, CANONIC Study Investigators of the EASL-CLIF Consortium (2013) Acute-onchronic liver failure is a distinct syndrome that develops in patients with acute decompensation of cirrhosis. Gastroenterology 144(7):14261437. https://doi.org/10.1053/j.gastro.2013.02.042

4. Li H, Chen LY, Zhang NN, Li ST, Zeng B, Pavesi M et al (2016) Characteristics, diagnosis and prognosis of acute-on-chronic liver failure in cirrhosis associated to hepatitis B. Sci Rep 6(1):1-14

5. Lopes, D., \& Samant, H. (2020). Hepatic Failure. In StatPearls. StatPearls Publishing.

6. Shah NJ, Mousa OY, Syed K, John S (2020) Acute on chronic liver failure. StatPearls Publishing, In StatPearls 
7. Clària J, Stauber RE, Coenraad MJ, Moreau R, Jalan R, Pavesi M, Amorós À, Titos E, Alcaraz-Quiles J, Oettl K, Morales-Ruiz M, Angeli P, Domenicali M, Alessandria C, Gerbes A, Wendon J, Nevens F, Trebicka J, Laleman W, Saliba F, Welzel TM, Albillos A, Gustot T, Benten D, Durand F, Ginès P, Bernardi M, Arroyo $V$, for the CANONIC Study Investigators of the EASL-CLIF Consortium and the European Foundation for the Study of Chronic Liver Failure (EFCLIF) (2016) Systemic inflammation in decompensated cirrhosis: characterization and role in acute-on-chronic liver failure. Hepatology 64(4): 1249-1264. https://doi.org/10.1002/hep.28740

8. Sole C, Sola E, Morales-Ruiz M, Fernàndez G, Huelin P, Graupera I, Moreira R, de Prada G, Ariza X, Pose E, Fabrellas N, Kalko S, Jiménez W, Ginès P (2016) Systemic inflammatory response profile in acute-on-chronic liver failure and its relationship with prognosis. J Hepatol 64(2):S445. https://doi.org/10.1016/ S0168-8278(16)00736-4

9. Alam A, Suen KC, Ma D (2017) Acute-on-chronic liver failure: recent update. J Biomed Res 31(4):283-300. https://doi.org/10.7555/JBR.30.20160060

10. Reddy MS, Rajalingam R, Rela M (2015) Liver transplantation in acute-onchronic liver failure: lessons learnt from acute liver failure setting. Hepatol Int 9(4):508-513. https://doi.org/10.1007/s12072-014-9603-z

11. Moreau, R. (2016, May). The pathogenesis of ACLF: the inflammatory response and immune function. In Seminars in liver disease (Vol. 36, No. 02, pp. 133-140). Thieme Medical Publishers.

12. Gustot T, Fernandez J, Garcia E, Morando F, Caraceni P, Alessandria C, Laleman W, Trebicka J, Elkrief L, Hopf C, Solís-Munoz P, Saliba F, Zeuzem S, Albillos A, Benten D, Montero-Alvarez JL, Chivas MT, Concepción M, Córdoba J, McCormick A, Stauber R, Vogel W, de Gottardi A, Welzel TM, Domenicali M, Risso A, Wendon J, Deulofeu C, Angeli P, Durand F, Pavesi M, Gerbes A, Jalan R, Moreau R, Ginés P, Bernardi M, Arroyo V, for the CANONIC Study Investigators of the EASL-CLIF Consortium, Aguilar-Melero P, Bañares R, Bocci M, Catalina MV, Chin JL, Coenraad MJ, Coilly A, Dorn L, Gatta A, Gerber L, Grøenbæk H, Graupera I, Guevara M, Hausen A, Karlsen S, Lohse AW, Maggioli C, Markwardt D, Martinez J, Marzano A, de la Mata García M, Mesonero F, Mookerjee RP, Moreno C, Morrell B, Mortensen C, Nevens F, Peck-Radosavljevic M, Rizzetto M, Romano A, Samuel D, Sauerbruch T, Simon-Talero M, Solà E, Soriano G, Sperl J, Spindelboeck W, Steib C, Valla D, Verbeke L, van Vlierberghe H, Wege H, Willars C, Baenas MY, Zaccherini G (2015) Clinical course of acute-on-chronic liver failure syndrome and effects on prognosis. Hepatology 62(1):243-252. https://doi. org/10.1002/hep.27849

13. Omran D, Alboraie M, Zayed RA, Wifi MN, Naguib M, Eltabbakh M, Abdellah M, Sherief AF, Maklad S, Eldemellawy HH, Saad OK, Khamiss DM, Kassas ME (2018) Towards hepatitis C virus elimination: Egyptian experience, achievements and limitations. World J Gastroenterol 24(38):4330-4340. https://doi.org/10.3748/wjg.v24.i38.4330

14. Elgharably A, Gomaa Al, Crossey MM, Norsworthy PJ, Waked I, TaylorRobinson SD (2017) Hepatitis C in Egypt-past, present, and future. Int J General Med 10:1

15. Fernández J, Acevedo J, Wiest R, Gustot T, Amoros A, Deulofeu C, Reverter E, Martínez J, Saliba F, Jalan R, Welzel T, Pavesi M, Hernández-Tejero M, Ginès $P$, Arroyo V, European Foundation for the Study of Chronic Liver Failure (2018) Bacterial and fungal infections in acute-on-chronic liver failure: prevalence, characteristics and impact on prognosis. Gut 67(10):1870-1880. https://doi.org/10.1136/gutjnl-2017-314240

16. Barosa R, Roque-Ramos L, Patita M, Nunes G, Fonseca J (2017) CLIF-C ACLF score is a better mortality predictor than MELD, MELD-Na and CTP in patients with Acute on chronic liver failure admitted to the ward. Rev Esp Enferm Dig 109(6):399-405. https://doi.org/10.17235/ reed.2017.4701/2016

17. Hernaez R, Solà E, Moreau R, Ginès $P$ (2017) Acute-on-chronic liver failure: an update. Gut 66(3):541-553. https://doi.org/10.1136/gutjnl-2016-312670

18. Arvaniti V, D'Amico G, Fede G, Manousou P, Tsochatzis E, Pleguezuelo M, Burroughs AK (2010) Infections in patients with cirrhosis increase mortality four-fold and should be used in determining prognosis. Gastroenterology 139(4):1246-1256. https://doi.org/10.1053/j.gastro.2010.06.019

19. Fernández J, Gustot T (2012) Management of bacterial infections in cirrhosis. J Hepatol 56:S1-S12. https://doi.org/10.1016/S0168-8278(12)60002-6

20. Propst-Graham KL, Preheim LC, Vander Top EA, Snitily MU, Gentry-Nielsen MJ (2007) Cirrhosis-induced defects in innate pulmonary defenses against Streptococcus pneumoniae. BMC Microbiol 7(1):1-10

21. Shi Y, Yang $Y$, Hu Y, Wu W, Yang $Q$, Zheng $M$, Zhang S, Xu Z, Wu Y, Yan H, Chen $Z$ (2015) Acute-on-chronic liver failure precipitated by hepatic injury is distinct from that precipitated by extrahepatic insults. Hepatology 62(1): 232-242. https://doi.org/10.1002/hep.27795

22. Piano S, Bartoletti M, Tonon M, Baldassarre M, Chies G, Romano A, Viale P, Vettore E, Domenicali M, Stanco M, Pilutti C, Frigo AC, Brocca A, Bernardi M, Caraceni P, Angeli P (2018) Assessment of Sepsis-3 criteria and quick SOFA in patients with cirrhosis and bacterial infections. Gut 67(10):1892-1899. https://doi.org/10.1136/gutjnl-2017-314324

23. Zhang Y, Li F, Miao L, Sun H, Bao X, Zhang D (2017) Establishment of a new acute-on-chronic liver failure model. Acta Pharmaceutica Sinica B 7(3):326-333

24. Ferrarese A, Feltracco P, Barbieri S, Cillo U, Burra P, Senzolo M (2019) Outcome of critically ill cirrhotic patients admitted to the ICU: the role of ACLF. J Hepatol 70(4):801-803. https://doi.org/10.1016/j.jhep.2018.09.015

25. Cervoni JP, Thévenot T, Weil D, Muel E, Barbot O, Sheppard F, Monnet E, di Martino V (2012) C-reactive protein predicts short-term mortality in patients with cirrhosis. J Hepatol 56(6):1299-1304. https://doi.org/10.1016/j.jhep.2 011.12 .030

26. Pieri G, Agarwal B, Burroughs AK (2014) C-reactive protein and bacterial infection in cirrhosis. Ann Gastroenterol 27(2):113

27. Chen BH, Tseng HJ, Chen WT, Chen PC, Ho YP, Huang CH, Lin CY (2020) Comparing eight prognostic scores in predicting mortality of patients with acute-on-chronic liver failure who were admitted to an ICU: a single-center experience. J Clin Med 9(5):1540. https://doi.org/10.3390/jcm9051540

28. Engelmann C, Thomsen KL, Zakeri N, Sheikh M, Agarwal B, Jalan R, Mookerjee RP (2018) Validation of CLIF-C ACLF score to define a threshold for futility of intensive care support for patients with acute-on-chronic liver failure. Crit Care 22(1):1-8

29. Ramzan M, lqbal A, Murtaza HG, Javed N, Rasheed G, Bano K (2020) Comparison of CLIF-C ACLF score and MELD score in predicting ICU mortality in patients with acute-on-chronic liver failure. Cureus 12(2):e7087. https://doi.org/10.7759/cureus.7087.

\section{Publisher's Note}

Springer Nature remains neutral with regard to jurisdictional claims in published maps and institutional affiliations.

\section{Submit your manuscript to a SpringerOpen ${ }^{\circ}$ journal and benefit from:}

- Convenient online submission

- Rigorous peer review

- Open access: articles freely available online

High visibility within the field

- Retaining the copyright to your article

Submit your next manuscript at $>$ springeropen.com 